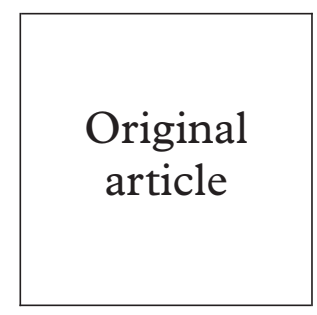

\title{
The Gay Men's Task Force: the impact of peer education on the sexual health behaviour of homosexual men in Glasgow
}

\author{
Lisa M Williamson, Graham J Hart, Paul Flowers, Jamie S Frankis, Geoff J Der
}

Objective: To assess the impact of a peer education intervention, based in the "gay" bars of Glasgow, which sought to reduce sexual risk behaviours for HIV infection and increase use of a dedicated homosexual men's sexual health service, and in particular increase the uptake of hepatitis $B$ vaccination.

Design: Self completed questionnaires administered to men who have sex with men (MSM) in Glasgow's gay bars.

Subjects: 1442 men completed questionnaires in January 1999, 7 months after the end of the 9 month sexual health intervention.

Main outcome measures: Self reported contact with the peer education intervention, reported behaviour change, and reported sexual health service use.

Results: The Gay Men's Task Force (GMTF) symbol was recognised by $42 \%$ of the men surveyed. Among men who reported speaking with peer educators $49 \%$ reported thinking about their sexual behaviour and $26 \%$ reported changing their sexual behaviour. Logistic regressions demonstrated higher levels of HIV testing, hepatitis B vaccination, and use of sexual health services among men who reported contact with the intervention. These men were more likely to have used the homosexual specific sexual health service. Peer education dose effects were suggested, with the likelihood of HIV testing, hepatitis B vaccination, and use of sexual health services being greater among men who reported talking to peer educators more than once.

Conclusion: The intervention had a direct impact on Glasgow's homosexual men and reached men of all ages and social classes. Higher levels of sexual health service use and uptake of specific services among men who had contact with the intervention are suggestive of an intervention effect. Peer education, as a form of health outreach, appears to be an effective intervention tool in terms of the uptake of sexual health services, but is less effective in achieving actual sexual behaviour change among homosexual men.

(Sex Transm Inf 2001;77:427-432)

Keywords: homosexual men; peer education; sexual behaviour; sexual health service use

\section{Introduction}

In 1999 there was an $11 \%$ increase in prevalent diagnosed HIV infection in homosexual men in the United Kingdom compared with 1998 (from 9637 to 10650 ). ${ }^{1}$ There is therefore continuing interest in finding successful means of preventing new infections. Peer education has been identified as a promising and popular form of intervention, ${ }^{2}$ and has been demonstrated to encourage the uptake of safer sex behaviours among homosexual men in the United States..$^{3-7}$ In Scotland over the past decade, sex between men was the primary route of HIV transmission, ${ }^{8}$ and previous research has found extensive sexual risk taking among this group, with a third of homosexual men in Scotland reporting recent unprotected anal intercourse. ${ }^{9}$ With the continuation of the HIV epidemic among homosexual men, and the apparent success of community level approaches in the United States, ${ }^{10}$ a number of agencies in Glasgow combined their preventive efforts to form the Gay Men's Task Force (GMTF). ${ }^{11}$ This evidence based, community level HIV prevention initiative employed peer educators to operate in "gay" bars to encourage homosexual men to reduce their sexual risk behaviour for HIV infection and increase their use of sexual health services, in particular the uptake of hepatitis B vaccination. Peer educators have not previously been employed to promote the uptake of sexual health services but, in a situation of low rates of vaccination among a population at high risk of the hepatitis B virus, ${ }^{912}$ this was considered a key element in the delivery of health promoting messages to men visiting Glasgow's gay bars.

Community level interventions do not require all individuals to have direct contact with the intervention, if they are based, as here, on the "diffusion of innovation" model. ${ }^{13}$ In this model it is expected that only a proportion of the intervention's impact will result from direct contact. The intervention will have further effects on the wider community as men who had contact with it converse and interact with other men. The wider community level effects of the intervention are reported elsewhere. ${ }^{14}$ This paper compares the experiences of those individuals who did report direct contact with the intervention and, in particular, direct contact with peer educators, with those who reported no intervention contact. We report on the efficacy of peer education in encouraging sexual behaviour change in gay bar patrons, 
and of their take up of sexual health services. We believe that this is the first study to evaluate peer education in terms of the wider sexual health needs of homosexual men, focusing on the potential role of peer educators as health outreach workers, with the aim of increasing the visibility and use of sexual health services.

\section{Methods}

The GMTF initiative trained 42 peer educators to work in bars contacting homosexual men. The peer educators wore distinctive uniforms (T-shirts, jackets, and bags) and, on entering the bars, would distribute GMTF leaflets on sexual health and behavioural issues and then approach men to discuss both these and wider issues along with advocating sexual health service uptake. ${ }^{15}$ During the 9 month intervention (October 1997 to June 1998) the peer educators reported 1484 contacts with men in all five of Glasgow's gay bars. A contact involved a conversation between a peer educator and a customer in the bar, where issues raised by both would be discussed and further leaflets distributed if required. The resultant discussions covered a wide range of health related topics, such as hepatitis $B$ vaccination and HIV testing, and while these did mainly reflect the content of the leaflets, other issues related to sexually transmitted infections and condoms and lubricants were also raised. ${ }^{16}$

Trained sessional research staff returned to the bars in January 1999 (7 months after the intervention) and asked men to self complete a short questionnaire. The questionnaire included general demographics, recent sexual behaviour, sexual health service use, and recollection of contact with the GMTF initiative.

In this paper we use recognition of the GMTF symbol, knowledge of the meaning of the GMTF acronym, and self reported contact with peer educators as contact indicators. A rising scale of contact is implied by the contact indicators. Recognition of the GMTF symbol could be gained from having seen the peer educators and the intervention literature in the bars without personally having any direct contact with it-the minimum level of contact. Knowledge of the acronym would require more involvement, such as observing this on the GMTF literature and uniforms or having read the GMTF leaflets-the intermediate level of contact. Self reported contact with a peer educator would require having talked to a peer educator-the maximum level of contact. Possible dose effects in peer education contact were examined through the comparison of number of contacts. Contact was reported as "none/not sure," "yes, once," and "yes, more than once."

Self reported consideration of sexual behaviour and self reported changes to sexual behaviour as a result of peer education contact are reported. Differences between contact and non-contact men are assessed through the comparison of chosen sexual health measures. ${ }^{11}$ The sexual health measures employed were reported hepatitis B vaccination, HIV antibody testing, sexually transmitted infection (STI) in the previous year, and HIV test recency (including all of 1998 and the first month of 1999 - that is, 6 months of the intervention and 7 months post-intervention). Differences in sexual health service use in the previous year were also compared. The sexual behaviour measure was unprotected anal intercourse (UAI) with casual partners (to examine differences in sexual risk behaviour). The Pearson $\chi^{2}$ test was used for bivariate analyses and statistically significant differences are identified throughout. Logistic regression was used to produce odds ratios and assess their significance. Adjusted odds ratios are presented where necessary.

\section{Results}

DEMOGRAPHIC PROFILE

Questionnaires were completed by 1442 men who have sex with men (MSM), representing a response rate of $75 \%$. Half of the sample was aged between 26 and 36 years old, with a range of 18-70 years, a mean of 32.1 years, and a median of 31 years (SD 9.01). More than half of the sample $(54 \%)$ came from the higher socioeconomic classes (I and II), 41\% reported being educated to degree or postgraduate level, and $79 \%$ were currently employed. Most men $(73 \%)$ lived in the Glasgow postcode area. The sample was sexually active, with most men (97\%) reporting some sexual contact, and $75 \%$ reporting anal intercourse with at least one partner in the previous year. Unprotected anal intercourse (UAI) in the previous year was reported by $33 \%$ of the sample and $35 \%$ of these men reported that on some occasions this had been with casual partners. The majority of men were frequent visitors of the gay bars; $86 \%$ attended the bars at least two or three times a month.

Overall, $29 \%$ of men reported use of a sexual health clinic in the previous year. In this subgroup, $45 \%$ reported that this had been at the Steve Retson Project (the only "gay specific" sexual health clinic in the area), while $36 \%$ had visited another genitourinary medicine (GUM) clinic and $20 \%$ had visited both in the previous year. There were no age or social class differences in sexual health clinic use. Visits to the Steve Retson Project are shown in figure 1 . The project observed a significant increase in new clients after the GMTF intervention started. There were 156 new clients in the 12 month period before the intervention, 347 in the 12 months including it, and 264 in the 12 months after it $(\mathrm{p}<0.0005)$.

\section{EXTENT OF INTERVENTION CONTACT}

The GMTF symbol was recognised by 576 men $(42 \%)$ and $524(36 \%)$ correctly specified the words that constitute the GMTF acronym. Nearly a third (424 men, 32\%) reported talking with a GMTF peer educator on at least one occasion. Of these, $53 \%$ had spoken only once and $47 \%$ had spoken more than once. Among the subgroup of men who reported speaking to a peer educator, $49 \%$ reported having thought about their sexual behaviour and $26 \%$ reported having changed their sexual behaviour to some extent as a result of these discussions. 


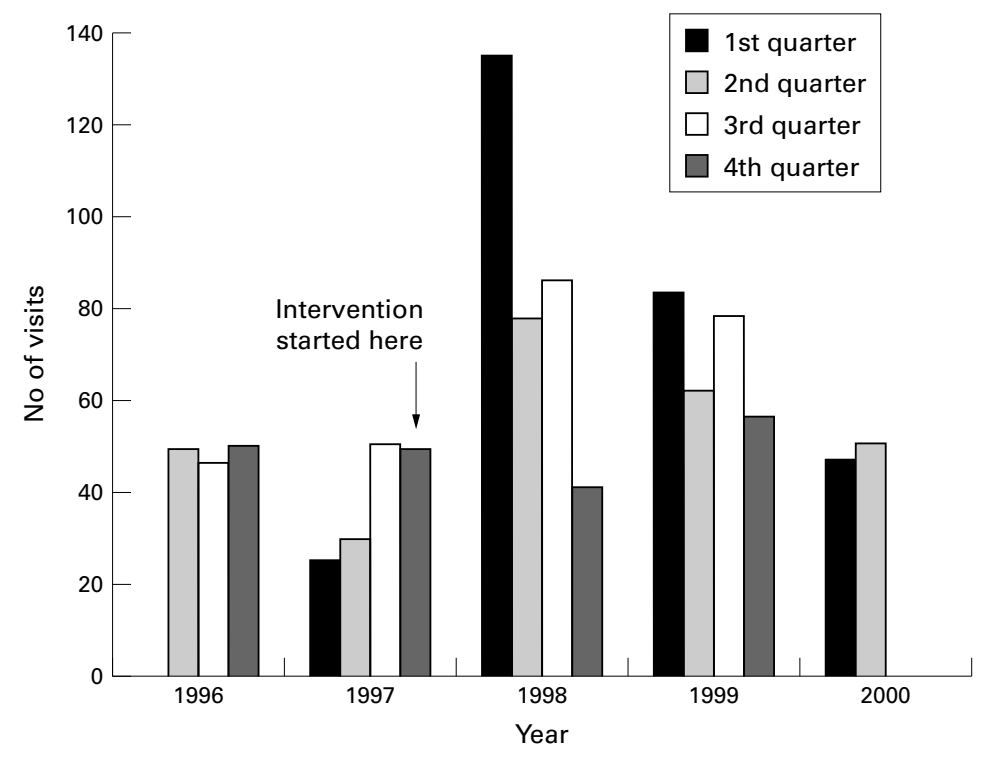

Figure 1 New visits to the Steve Retson Project (1 April 1996 to 30 fune 2000).

The demographic profiles of contact and non-contact men were very similar. There were no significant differences in GMTF contact between men in higher (social classes I, II, and IIIN) and lower (social classes IIIM, IV, and V) social classes, or between younger (15-25 years) and older (26 years and over) men.

Men who visited the bars more often were significantly more likely to report contact with GMTF. A linear trend was demonstrated by the Mantel-Haenszel $\chi^{2}$ test. The GMTF symbol was recognised by $54 \%$ of the most frequent visitors to the bars (four/five times a week) compared with $15 \%$ of the least frequent visitors (less than once a month) $(\mathrm{p}<0.0005)$. The meaning of the acronym was known by $47 \%$ of the most frequent visitors compared with $12 \%$ of the least frequent visitors $(\mathrm{p}<0.0005)$. Only $10 \%$ of the least frequent visitors reported talking to a peer educator compared with $48 \%$ of the most frequent visitors $(p<0.0005)$. However, men who reported contact with peer educators and attended bars frequently were no more likely to report having thought about or changed their sexual behaviour than men who reported contact with peer educators but made less frequent visits to the bars.

Because of the link found between the frequency of bar attendance and intervention contact the potential confounding effect of this variable was checked by examining its direct effect on the sexual health measures. Bar attendance did not have a significant effect on the likelihood of hepatitis B vaccination, HIV antibody testing, recency of HIV test, STI in the previous year, or UAI with casual partners. Therefore, frequency of bar attendance is not included in these analyses. It did have a significant effect on the likelihood of any GUM clinic use and adjusted odds ratios, controlling for frequency of bar attendance, are therefore presented. Similarly it was possible that reported experience of STI in the previous year would have a confounding effect on clinic use. Reporting STI in the previous year was found to have a significant effect on all four clinic use variables so adjusted odds ratios controlling for this are presented.

\section{CONTACT EFFICACY}

Odds ratios were calculated for contact and non-contact men with respect to the sexual health measures and significant variation was found (table 1). Contact men were significantly more likely to report each of the measures than non-contact men, except for UAI with casual partners and STI in the previous year (for men who knew the meaning of the acronym). Adjusted odds ratios (controlling for STI in the previous year) were calculated for clinic use and, with the exception of exclusive use of other GUM clinics, contact men were significantly more likely to report clinic use than non-contact men (table 1). Both experience of STI in the previous year and intervention contact were significant predictors of clinic use. This indicates the independent effects of STI $(p<0.01$ in each analysis) and each of the contact indicators on the sexual health measures (table 1).

With respect to use of the Steve Retson Project there was a significant interaction between contact with a peer educator and STI in the previous year. Use of this clinic was significantly more likely among those men who reported both $(\mathrm{OR}=3.69$, 95\% CI 1.17 11.62).

To examine the possibility that contact with GMTF leaflets would be enough to effect behaviour change the odds ratios were recalculated for men who recognised the GMTF symbol or knew the meaning of the acronym, but excluding those who also reported talking to peer educators. In these analyses, recognition

Table 1 Contact efficacy of Gay Men's Task Force (GMTF) intervention-comparison of contact and non-contact men (logistic regression)

\begin{tabular}{|c|c|c|c|c|c|c|}
\hline \multirow[b]{2}{*}{ Sexual health behaviour } & \multicolumn{2}{|c|}{ Recognise GMTF symbol } & \multicolumn{2}{|c|}{ Know meaning of GMTF acronym } & \multicolumn{2}{|c|}{ Talked to peer educator } \\
\hline & Odds ratio & $95 \% C I$ & Odds ratio & $95 \% C I$ & Odds ratio & $95 \% C I$ \\
\hline Hepatitis B vaccination & $1.49^{\star \star \star}$ & $1.20-1.85$ & $1.43^{\star \star}$ & $1.15-1.78$ & $1.66^{\star \star \star}$ & $1.32-2.11$ \\
\hline HIV antibody test & $1.42^{\star \star}$ & $1.15-1.77$ & $1.35^{\star \star}$ & $1.08-1.68$ & $1.32^{\star}$ & $1.04-1.66$ \\
\hline Recency of HIV test (previous year) & $1.37^{\star}$ & $1.05-1.77$ & $1.32^{\star}$ & $1.02-1.71$ & $1.43^{\star}$ & $1.09-1.88$ \\
\hline Sexually transmitted infections & $1.68^{\star}$ & $1.13-2.50$ & 1.46 & $0.98-2.17$ & $1.69^{\star}$ & $1.12-2.54$ \\
\hline UAI with casual partners $\dagger$ & 0.97 & $0.64-1.46$ & 0.82 & $0.54-1.23$ & 0.76 & $0.49-1.18$ \\
\hline Any GUM clinic use $\neq \int$ & $2.91^{\star \star \star}$ & $2.24-3.76$ & $2.83^{\star \star \star}$ & $2.19-3.65$ & $2.25^{\star \star \star}$ & $1.73-2.93$ \\
\hline Steve Retson Project only $\ddagger$ & $4.64^{\star \star \star}$ & $3.23-6.66$ & $4.39^{\star \star \star}$ & $3.11-6.21$ & $2.98^{\star \star \star}$ & $2.14-4.14$ \\
\hline Other GUM clinic only $\ddagger$ & 0.89 & $0.62-1.28$ & 0.89 & $0.61-1.28$ & 0.82 & $0.56-1.23$ \\
\hline Both Steve Retson and other GUM $\ddagger$ & $3.09^{\star \star \star}$ & $1.86-5.14$ & $2.91^{\star \star \star}$ & $1.78-4.74$ & $2.87^{\star \star \star}$ & $1.76-4.71$ \\
\hline
\end{tabular}

†Among men reporting UAI.

¥Adjusted odds controlling for experience of sexually transmitted infection in the previous year.

\Adjusted odds controlling for frequency of bar attendance.

${ }^{\star} \mathrm{p}<0.05,{ }^{\star \star} \mathrm{p}<0.01,{ }^{\star \star \star} \mathrm{p}<0.001$. 
Table 2 Contact efficacy of Gay Men's Task Force (GMTF) intervention-comparison of contact and non-contact men, excluding men who also reported talking to peer educators (logistic regression)

\begin{tabular}{|c|c|c|c|c|}
\hline \multirow[b]{2}{*}{ Sexual health behaviour } & \multicolumn{2}{|c|}{ Recognise GMTF symbol } & \multicolumn{2}{|c|}{ Know meaning of GMTF acronym } \\
\hline & Odds ratio & $95 \% C I$ & Odds ratio & $95 \% C I$ \\
\hline Hepatitis B vaccination & 1.15 & $0.84-1.57$ & 1.05 & $0.76-1.47$ \\
\hline HIV antibody test & 1.09 & $0.80-1.49$ & 1.00 & $0.72-1.38$ \\
\hline Recency of HIV test (previous year) & 1.05 & $0.72-1.54$ & 1.08 & $0.72-1.62$ \\
\hline Sexually transmitted infections & 1.42 & $0.79-2.55$ & 1.31 & $0.70-2.45$ \\
\hline UAI with casual partners $\dagger$ & 1.57 & $0.87-2.83$ & 1.43 & $0.77-2.65$ \\
\hline Any GUM clinic use $\neq \subseteq$ & $2.20^{\star \star \star}$ & $1.53-3.15$ & $2.21^{\star \star \star}$ & $1.52-3.20$ \\
\hline Steve Retson Project only $\ddagger$ & $4.21^{\star \star \star}$ & $2.60-6.79$ & $3.89^{\star \star \star}$ & $2.40-6.32$ \\
\hline Other GUM clinic only $\ddagger$ & 0.78 & $0.45-1.35$ & 0.82 & $0.46-1.44$ \\
\hline Both Steve Retson and other GUM $\ddagger$ & $2.49^{\star}$ & $1.17-5.28$ & $2.69^{\star}$ & $1.25-5.76$ \\
\hline
\end{tabular}

†Among men reporting UAI.

†Adjusted odds controlling for experience of sexually transmitted infection in the previous year.

\Adjusted odds controlling for frequency of bar attendance.

${ }^{\star} \mathrm{p}<0.05,{ }^{\star \star} \mathrm{p}<0.01,{ }^{\star \star \star}{ }^{\star} \mathrm{p}<0.001$.

of the symbol and knowledge of the acronym were no longer significant predictors of the sexual health measures but did still predict sexual health clinic use, with the exception of exclusive use of other GUM clinics (table 2).

PEER EDUCATION DOSE EFFECTS

Logistic regression was used to calculate odds ratios for peer education dose effects (table 3 ). Men who reported talking to a peer educator more than once were more likely than noncontact men to report each of the sexual health measures, except for more recent HIV tests. Among the subgroup of men reporting UAI, those who reported talking to a peer educator more than once were significantly less likely to report UAI with casual partners in the last year. Men who reported talking to a peer educator once were more likely just to report hepatitis B vaccination and more recent HIV tests than non-contact men. We again calculated adjusted odds for clinic use (controlling for STI in the previous year) and the likelihood of use was significantly higher for both groups of peer education contact men than for men who did not talk to peer educators (table 3). Experience of STI in the previous year also increased the likelihood of clinic use $(p<0.01$ in each analysis). Exclusive use of other GUM clinics was significantly higher among men with experience of STI in the previous year $(p<0.0005)$ but not among either group of peer education contact men. An interaction between talking to a peer educator once and experience of STI in the previous year was apparent, as men who reported this were significantly more likely than men who had not talked to a peer educator to have used the Steve Retson Project $(\mathrm{OR}=4.13,95 \%$ CI 1.10 15.60).

To examine the significance of these dose effects we compared men who had one contact with a peer educator with men who had more than one contact. Men who reported more than one contact were more likely than one contact men to be HIV tested (OR $=1.53,95 \%$ CI 1.04-2.26) and less likely to report UAI with casual partners $(\mathrm{OR}=0.41,95 \%$ CI $0.19-$ $0.91)$. The difference between one contact and more than one contact men was also significant for combined use of the Steve Retson Project and another GUM clinic, with the likelihood being higher for men with more than one contact $(\mathrm{OR}=2.05$, 95\% CI 1.04-4.04).

\section{Discussion}

The GMTF initiative had an impact on Glasgow's gay scene, with a third of men surveyed at the 7 month follow up reporting some form of contact with it. Most importantly, the intervention was not selective in relation to key sociodemographic variables, reaching men of all ages and social classes in the gay bars. The demographic profile of these men is comparable to that of other bar based samples. ${ }^{517}$

It is encouraging that this intervention reached men of all ages. Previous research has identified young homosexual men as being more likely to engage in high risk behaviour,

Table 3 Peer education dose effects-comparison of men who talked to peer educators on one or more than one occasion and men who did not talk to peer educators (logistic regression)

\begin{tabular}{|c|c|c|c|c|c|}
\hline \multirow{4}{*}{$\begin{array}{l}\text { Sexual health behaviour } \\
\text { Hepatitis B vaccination }\end{array}$} & \multicolumn{5}{|c|}{ Talked to a peer educator } \\
\hline & \multirow{2}{*}{\multicolumn{3}{|c|}{$\begin{array}{l}\text { Odds ratio }(95 \% \text { CI) } \\
\text { "No/not sure" "Yes, once" }\end{array}$}} & \multirow{2}{*}{\multicolumn{2}{|c|}{ "Yes, more than once" }} \\
\hline & & & & & \\
\hline & 1 & $1.61^{\star \star}$ & $(1.19-2.17)$ & $1.73^{\star \star \star}$ & $(1.26-2.37)$ \\
\hline HIV antibody test & 1 & 1.08 & $(0.80-1.45)$ & $1.65^{\star \star}$ & $(1.21-2.26)$ \\
\hline Recency of HIV test (previous year) & 1 & $1.44^{\star}$ & $(1.02-2.02)$ & 1.42 & $(0.99-2.03)$ \\
\hline Sexually transmitted infections & 1 & 1.50 & $(0.89-2.53)$ & $1.90^{\star}$ & $(1.14-3.16)$ \\
\hline UAI with casual partners $\dagger$ & 1 & 1.08 & $(0.64-1.84)$ & $0.44^{\star}$ & $(0.23-0.88)$ \\
\hline Any GUM clinic use $\neq$ & 1 & $1.95^{\star \star \star}$ & $(1.40-2.70)$ & $2.64^{\star \star \star}$ & $(1.88-3.70)$ \\
\hline Steve Retson Project only $\ddagger$ & 1 & $2.68^{\star \star \star}$ & $(1.79-4.01)$ & $3.33^{\star \star \star}$ & $(2.22-4.97)$ \\
\hline Other GUM clinic only‡ & 1 & 0.97 & $(0.60-1.56)$ & 0.67 & $(0.38-1.18)$ \\
\hline Both Steve Retson and other GUM $\ddagger$ & 1 & $1.94^{\star}$ & $(1.02-3.71)$ & $3.99^{\star \star \star}$ & $(2.28-6.98)$ \\
\hline
\end{tabular}

†Among men reporting UAI.

†Adjusted odds controlling for experience of STI in previous year.

\Adjusted odds controlling for frequency of bar attendance.

${ }^{\star} \mathrm{p}<0.05,{ }^{\star \star} \mathrm{p}<0.01,{ }^{\star \star \star} \mathrm{p}<0.001$. 
such as UAI, and to be less knowledgeable about safer sex. ${ }^{17}$ They have also been found to perceive less risk in UAI even while seeing themselves as being more at risk from AIDS. ${ }^{18}$ Lack of peer support for safer sex has also been identified as a predictor of UAI among young homosexual men. ${ }^{19}$ The lack of demographic difference in intervention effect suggests that peer education is a promising intervention for men from all age groups and social classes.

Approximately a third of Glasgow men reported UAI with casual partners in the preceding year, regardless of whether they reported contact with the intervention. This is similar to levels found previously in Scotland and England ${ }^{90} 21$ and higher than levels found in the United States. ${ }^{4}$ Among men reporting UAI, only men who reported speaking with peer educators more than once were less likely to report UAI with casual partners; this is despite the behaviour change reported by contact men in general. There appears therefore to be some HIV related risk reduction among men exposed to the larger peer education "dose." While HIV related risk reduction was limited sexual health awareness was higher among contact men in general, with higher levels of HIV antibody testing and hepatitis B vaccination. The peer educators reported it was easier to talk about sexual health than safer sex behaviour, ${ }^{15}$ and this may account for the failure of the intervention to effect sexual behaviour change, but also its success in increasing the uptake of sexual health services. When men who talked to peer educators were excluded from the analysis recognition of the GMTF symbol and knowledge of the acronym were no longer significant predictors of HIV testing or hepatitis B vaccination. This suggests that actual contact with a peer educator was necessary in the promotion of these services.

The higher level of STI among men who reported talking to a peer educator does not necessarily constitute a negative outcome. A higher incidence of STI, combined with greater use of sexual health services among men who had direct contact with the intervention, could be indicative of a positive outcome if the increase is associated with more diagnoses and treatment because of increased service use. Detection of asymptomatic (for example, Chlamydia trachomatis) or, for patients, difficult to recognise (for example, genital warts) STI is also a possible and positive outcome. Increased awareness of sexual health issues and the early detection and treatment of STI could have a longer term positive effect on incidence if the total number of prevalent infections is reduced in this sexually active population.

Men who had any contact with the intervention reported higher levels of sexual health service use. This was particularly the case with respect to the Steve Retson Project, the gay specific sexual health service in Glasgow, and one of the agencies central to the intervention. Indeed, peer educators and GMTF leaflets specifically promoted this service above others and the project ran an extra clinic as part of the intervention. The project observed a significant increase in new clients after the intervention started and the increase was maintained after the intervention ended. Men who reported talking to a peer educator were most likely to have used the Steve Retson Project, particularly if they also reported STI in the previous year. The peer educators also reported increasing acceptance of visiting the Steve Retson Project among men in the bars during the intervention period. ${ }^{15}$

Men who reported talking to peer educators more than once were most likely to report hepatitis B vaccination, HIV antibody testing, and sexual health service use. This is suggestive of a peer education dose effect. We found that the dose effect, the difference between one and more than one contact, was significant with regard to HIV testing, UAI with casual partners and combined use of the Steve Retson Project and another GUM clinic. Such dose effects have been reported previously by Kelly et al in relation to sexual risk behaviour, ${ }^{22}$ but not in their most recent work. ${ }^{5}$ Kegeles et al make no mention of dose effects on sexual behaviour with regard to their community based intervention for young men. ${ }^{4}$ We are aware of no other research with regard to dose effects of peer education on the uptake of sexual health services.

It is not possible for us to conclude that the differences found between contact and noncontact men would not have occurred anyway or were not in fact already present. However, men who had contact with the intervention reported higher levels of sexual health service use and men who had contact with peer educators reported higher levels of recent HIV tests. Combined with the peer education dose effects and the increase in clients at the Steve Retson Project, this lends support to at least some level of intervention effect. This could, however, be the result of reverse causation, in that men who are more aware of sexual health issues may have been more receptive to the intervention and, in particular, to contact with peer educators. The concern that only men who have higher levels of sexual health awareness will take part in individual and small group interventions could also apply to community level interventions. ${ }^{10}$

This is the first paper to report the impact of peer education on the uptake of sexual health services, and to demonstrate an increase in service use as a result of a targeted intervention. It should be noted that only men who visit Glasgow's gay bars would have had the opportunity of contact with the intervention. This limitation is countered by the possibility that it is these men who are most in need of such an intervention, given the high levels of risk behaviour identified in this population. ${ }^{9}$

These results suggest that peer education is effective in increasing sexual health awareness and the uptake of sexual health services among men having direct contact with the intervention. Levels of sexual health service use were higher among men who reported any contact with the intervention, levels of particular service uptake were higher among men who reported direct contact with peer educators, and sexual risk behaviour was only lower 
among men who reported multiple contact with peer educators. If the differences between contact and non-contact men were the result of the intervention it may be that a longer term community level effect will become apparent. Men who have used sexual health services could advise others (not directly touched by the intervention) to do so, as posited by "diffusion of innovation" theory. ${ }^{13}$ However, it is in relation to sexual behaviour change that peer education was first introduced, and it is clear that continuing sexual risk behaviour among Glasgow's homosexual men requires further targeted prevention strategies if reductions in this behaviour are to be achieved. ${ }^{23}$ Peer education has been shown in this paper to be successful for sexual health service outreach, but its effect on sexual behaviour has yet to be demonstrated in the United Kingdom.

This research was funded by the UK Medical Research Council.

We would like to acknowledge the role of staff at the Steve Retson Project including Dr Richard Hillman, John Roberts, and Chris Thow, and staff at Phace West including John Wilkes and Chris Thow, and staff at Phace West including John Wilkes and Patrick Stoakes; and the support of Body Positive Strathclyde, Strathclyde Lesbian and Gay Switchboard, and Glasgow Gay and Lesbian Centre for their contribution to the
Gay Men's Task Force. The peer educators, who number too Gay Men's Task Force. The peer educators, who number too
many to list, are thanked for their hard work and commitment to
the GMTF. Claire Marriott was involved in baseline, process the GMTF. Claire Marriott was involved in baseline, process and outcome research associated with this project. Finally, bar managers, staff, and most importantly their customers in Glasgow are due our deepest gratitude for their willingness to complete questionnaires and participate in the GMTF.

thank the referee for valuable comments on the paper.

Contributions: LW undertook analysis of data and wrote the first draft, and contributed to subsequent drafts, of the paper; $\mathrm{GH}$ initiated and designed the study and wrote second, and revised subsequent, drafts of the paper; PF contributed to the design of the study, training of peer educators and to drafts of the paper; JF trained peer educators, collected data, and contributed to the writing of the paper; GD provided statistical contributed to the writing of the paper; GD provided statistical data.

1 CDSC. Further increase in prevalent diagnosed HIV in the United Kingdom 1999. Commun Dis Rep CDR Weekly 2000;10:265-8.

2 Hart G. Peer education and community based HIV prevenHart G. Peer education and community based HIV preven-
tion for homosexual men: peer led, evidence based, or fashtion for homosexual men: peer led, evidence

3 Kegeles SM, Hays RB, Coates TJ. The Mpowerment Project: a community-level HIV prevention intervention for young gay men. Am f Public Health 1996;86:1129-36.
4 Kegeles SM, Hays RB, Pollack LM, et al. Mobilizing young gay and bisexual men for HIV prevention: a twocommunity study. AIDS 1999;13:1753-62.

5 Kelly JA, Murphy DA, Sikkema KJ, et al. Randomised, controlled, community-level HIV-prevention intervention for sexual-risk behaviour among homosexual men in US cities. Lancet 1997;350:1500-5.

6 Kelly JA, St. Lawrence JS, Stevenson LY, et al. Community AIDS/HIV risk reduction: the effects of endorsements by popular people in three cities. Am F Public Health 1992;82: 1483-9.

7 Kelly JA, St Lawrence JS, Diaz YE, et al. HIV risk behaviour reduction following intervention with key opinion leaders of population: an experimental analysis. Am $\mathcal{F}$ Public Health of population: an

8 SCIEH. ANSWER HIV Infection and AIDS: Quarterly report to 30 June 1999. SCIEH Weekly Report 1999;33(99/
reflerty 28): $178-85$

9 Hart GJ, Flowers P, Der GJ, et al. Homosexual men's HIV related sexual risk behaviour in Scotland. Sex Transm Inf 1999;75:242-6.

10 Kegeles S, Hart G. Recent HIV prevention interventions for gay men: individual, small-group and community-based studies. AIDS 1998;12(suppl A):S209-15.

11 Flowers P, Frankis J, Hart G. Evidence and the evaluation of a community-level intervention: researching the Gay Men's Task Force Initiative. In Watson J, Platt S, eds. Researching health promotion. London: Routledge, 2000.

12 Hart G, Dawson J, Fitzpatrick R, et al. Risk behaviour, anti HIV and anti HBc prevalence in clinic and non-clinic samples of gay men in England 1991-2. AIDS 1993;7:863-9.

13 Rogers EM. Diffusion of innovations. 2nd ed. New York: Free Press, 1983.

14 Flowers P, Hart GJ, Williamson LM, Frankis JS, et al. Does bar-based, peer-led sexual health promotion have a community-level effect amongst gay men in Scotland? Int $\mathcal{F}$ STD AIDS (in press).

15 Flowers P, Frankis J, Hart G. Experiential aspects of peer education in gay bars. (In press).

16 Frankis J, Flowers P, Hart G. Gay Men's Task Force: preliminary evaluation of service delivery. Glasgow: MRC Medical nary evaluation of service delivery. Glasgow:
Sociology Unit, 1998 . Working Paper No 68.

17 Kelly JA, St Lawrence JS, Brasfield TL, et al. Psychological factors that predict AIDS high-risk versus AIDS precauionary behaviour. 7 Consult Clinical Psychol 1990;58:11720.

18 Hays R, Kegeles S, Coates T. High HIV risk-taking among young gay men. AIDS 1990;4:901-7.

9 Lemp GF, Hirozawa AM, Givertz D, et al. Seroprevalence of HIV and risk behaviours among young homosexual and bisexual men: the San Francisco/Berkeley young men's survey. FAMA 1994;272:449-54.

20 Dodds J, Nardone A, Mercey DE, et al. Increase in high risk sexual behaviour among homosexual men, London 1996-8: cross sectional, questionnaire study. BMf 2000; 320:1510-1

21 Davies PM, Hickson FCI, Weatherburn P, et al. Sex, gay men and AIDS. London: Falmer Press, 1993.

22 Kelly JA, Winett RA, Roffman RE, et al. Social diffusion models can produce population HIV-risk behaviour models can produce population HIV-risk behaviour reduction: field trial results and mechanisms underlying STD World Congress. Berlin, Germany, 1993.

23 Coates TJ, Aggleton P, Gutzwiller F, et al. HIV prevention in developed countries. Lancet 1996;348:1143-8. 